\title{
Entangled quantum probes for dynamical environmental noise
}

\author{
Matteo A. C. Rossi* and Matteo G. A. Paris ${ }^{\dagger}$ \\ Dipartimento di Fisica, Università degli Studi di Milano, 20133 Milano, Italy
}

(Received 17 March 2015; published 28 July 2015)

\begin{abstract}
We address the use of entangled qubits as quantum probes to characterize the noise induced by complex environments. In particular, we show that a joint measurement on entangled probes can improve estimation of the correlation time for a broad class of environmental noises compared to sequential strategies involving single-qubit preparation. The enhancement appears when the noise is faster than a threshold value, a regime which may always be achieved by tuning the coupling between the quantum probe and the environment inducing the noise. Our scheme exploits time-dependent sensitivity of quantum systems to decoherence and does not require dynamical control on the probes. We derive the optimal interaction time and the optimal probe preparation, showing that it corresponds to multiqubit Greenberger-Horne-Zeilinger states when entanglement is useful. We also show the robustness of the scheme against depolarization or dephasing of the probe, and discuss simple measurements approaching optimal precision.
\end{abstract}

DOI: 10.1103/PhysRevA.92.010302

PACS number(s): 03.67.Bg, 03.65.Yz, 03.67.-a, 05.40.-a

The coherence properties of a quantum system are strongly affected by its interaction with the surrounding environment. This is often an obstacle to the implementation of quantum technologies, so that much effort has been devoted to study the system-environment interaction and to engineer decoherence in order to minimize its degrading effects $[1,2]$. On the other hand, the very sensitivity of quantum systems to external influences also provides an effective tool to characterize unknown parameters of a given environment $[3,4]$ by exploiting quantum probes, as opposed to classical ones, usually macroscopic and more intrusive. Indeed, characterizing the noise induced by an external complex system is of great relevance in many areas of nanotechnology, as well as in monitoring biological or chemical processes [5-8]. Besides, it represents a crucial step in designing robust quantum protocols resilient to noise [9-14].

The proper framework to address characterization by quantum probes [15,16], and to design the best working conditions, is given by quantum estimation theory [17], which provides analytical tools to optimize the three building blocks of an estimation strategy: (i) preparation of the probe system in a suitably optimized state, (ii) controlled interaction of the probe with the system for an optimal amount of time $t$, and (iii) measurement of an optimal observable on the probe. Overall, the ultimate precision for any unbiased estimator $\hat{\gamma}$ of a certain parameter $\gamma$ is bounded by the quantum Cramèr-Rao (CR) theorem, stating that $\operatorname{Var}(\hat{\gamma}) \geqslant[M H(\gamma)]^{-1}$, where $M$ is the number of measurements and $H(\gamma)$ is the quantum Fisher information (QFI), i.e., the superior of the Fisher information over all possible quantum measurements described by positive operator-valued measures (POVMs).

Recently, single-qubit quantum probes have been proposed for the characterization of noise by monitoring decoherence and dephasing induced by the environment under investigation, in particular, when the latter can be described in terms of classical stochastic processes [18-22]. Indeed, stochastic modeling of the environment has been proven [23] to reliably

\footnotetext{
*matteo.rossi@unimi.it

†matteo.paris@ fisica.unimi.it
}

describe noise and the decoherence process in several systems affected by dephasing [24-31]. It may also be useful for several other systems of interest, including motional averaging [32] and solid-state qubits [33-35].

In this Rapid Communication, we extend this analysis to entangled qubits used as quantum probes, and show how they greatly improve the characterization of a broad class of environmental noises compared to other sequential strategies involving single-qubit preparation [36-38]. In particular, we show how to improve estimation of the correlation time (i.e., the spectral width) of classical noise. Since such noise is usually emerging from a large collection of fluctuators, we are going to consider Gaussian stochastic processes.

The probing scheme is depicted in Fig. 1, both for a sequence of $N$ uncorrelated qubits and for an $N$-qubit entangled state. In both cases we assume that the qubits do not interact with each other. In each case, the qubits may interact with different realizations of the noise or with the same realization, depending on the temporal and spatial distance between the probes. We end up with four possible schemes, which we describe in detail in the Supplemental Material [39]. In the following, we focus on the best case for each configuration, i.e., independent realizations for the separable probes and a common environment for entangled probes.

Let us start by considering a single qubit interacting with a fluctuating dephasing environment. The Hamiltonian is given by $\mathcal{H}(t)=\omega_{0} \sigma_{z}+B(t) \sigma_{z}$, where $\omega_{0}$ is the energy of the qubit and $B(t)$ is a realization of the stochastic process that describes the noise. As a paradigmatic example we consider a zero-mean Ornstein-Uhlenbeck process characterized by the autocorrelation function $K\left(t, t^{\prime}\right) \equiv\left\langle B(t) B\left(t^{\prime}\right)\right\rangle_{B}=\frac{1}{2} \gamma \Gamma \exp \left[-\gamma\left|t-t^{\prime}\right|\right]$, or by the corresponding Lorentzian spectrum. Here, $\gamma$ is the spectral width, i.e., the inverse of the autocorrelation time, while $\Gamma$ denotes the coupling between the probe and the system. It is worth noticing that a similar analysis may be carried out for other Gaussian processes, e.g., processes with power-law or Gaussian autocorrelation functions, and that results are qualitatively the same, independently on the choice of the autocorrelation function. 

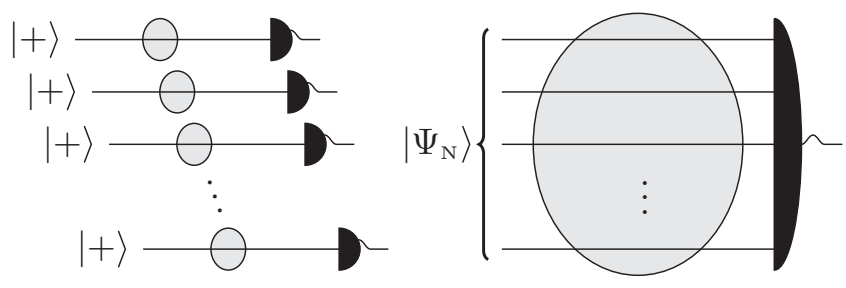

FIG. 1. Schematic diagram of possible characterization techniques. On the left, we have $N$ qubits, and each one is prepared in the state $|+\rangle$, then interacts with the system for a time $t$, and is finally measured independently of the other qubits. On the right, the $N$ qubits are initially prepared in a multiqubit Greenberger-Horne-Zeilinger (GHZ) state $\left|\Psi_{\mathrm{N}}\right\rangle$, and are allowed to interact with the system such that they are subject to the same realization of the noise. At the output, a collective measurement is performed on the qubits.

The density operator of the evolved qubit is given by

$$
\rho(t)=\left\langle U(t) \rho(0) U^{\dagger}(t)\right\rangle_{B}
$$

where $\langle\cdot\rangle_{B}$ denotes the average over all possible realizations of the stochastic process in the time interval $[0, t], U(t)=$ $\exp \left[-i \int_{0}^{t} \mathcal{H}(s) d s\right]=\exp \left\{\left[-i \omega_{0} t+\phi(t)\right] \sigma_{z}\right\}$ is the time evolution operator, and $\phi(t)=\int_{0}^{t} B(s) d s$ is the accumulated phase due to the environmental (dynamical) noise. An explicit expression for $\rho(t)$ can be found by employing the characteristic function of a zero-mean Gaussian stochastic process, $\left\langle e^{i m \phi(t)}\right\rangle=e^{-\frac{1}{2} m^{2} \beta_{\gamma}(t)}$, where

$$
\beta_{\gamma}(t)=\int_{0}^{t} \int_{0}^{t} d s d w K(s, w)=\frac{\Gamma}{\gamma}\left(e^{-\gamma t}+\gamma t-1\right) .
$$

If the qubit is initially prepared in a state described by the density operator $\rho(0)$, the density operator at the time $t$ will be $\rho(t)$ with $\rho_{k k}(t)=\rho_{k k}(0), k=1,2$, and

$$
\rho_{12}(t)=e^{-2\left[i \omega_{0} t+\beta_{\gamma}(t)\right]} \rho_{12}(0) .
$$

The optimal single-qubit preparation, given by $|+\rangle=$ $\frac{1}{\sqrt{2}}(|0\rangle+|1\rangle)$, together with the corresponding QFI and the optimal measurement for the estimation of $\gamma$, have been recently found [22]. For $N$ uncorrelated qubits, owing to additivity, the QFI is just $N$ times the single-qubit QFI, i.e.,

$$
H_{\mathrm{N}}^{\mathrm{SEP}}(\gamma, t)=\frac{4 N}{e^{4 \beta_{\gamma}(t)}-1}\left[\partial_{\gamma} \beta_{\gamma}(t)\right]^{2} .
$$

Let us now consider a probe made of $N$ qubits initially prepared in the generalized GHZ entangled state $\left|\Psi_{\mathrm{N}}\right\rangle=$ $\left(|0\rangle^{\otimes N}+|1\rangle^{\otimes N}\right) / \sqrt{2}$, interacting with a common environment. We also assume that the qubits do not interact with each other and that they interact with a common environment, i.e., with the same realization of the noise. The overall Hamiltonian is thus

$$
\mathcal{H}^{(\mathrm{N})}(t)=\mathcal{H}(t) \otimes \mathbb{I}^{\otimes N-1}+\mathbb{I} \otimes \mathcal{H}(t) \otimes \mathbb{I}^{\otimes N-2}+\cdots,
$$

where $\mathcal{H}(t)$ is the above single-qubit Hamiltonian and $B(t)$ is the same realization of the noise for all the qubits. The QFI for the parameter $\gamma$ reads

$$
H_{\mathrm{N}}^{\mathrm{GHZ}}(\gamma, t)=\frac{4 N^{4}}{e^{4 N^{2} \beta(t, \gamma)}-1}\left[\partial_{\gamma} \beta_{\gamma}(t)\right]^{2} .
$$
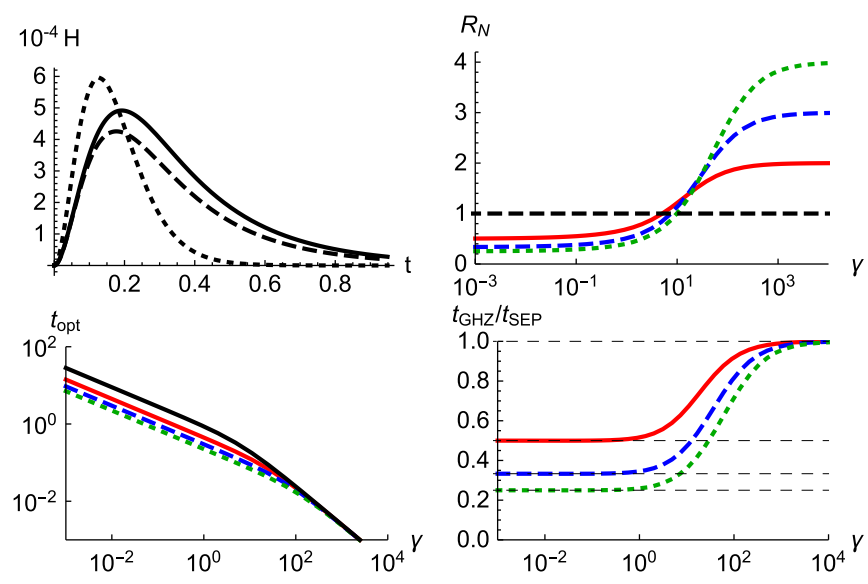

FIG. 2. (Color online) In the upper left panel, the QFI $H_{2}^{\mathrm{SEP}}(t)$ (solid) and $H_{2}^{\mathrm{GHZ}}(t)$ (dotted) as a function of time for $\gamma=10$. We also show for comparison the (smaller) QFI for separable two-qubit probes in a common environment (dashed). In the upper right the QFI ratio $R_{\mathrm{N}}$ as a function of $\gamma$ (log scale) for $N=2$ (solid red), $N=3$ (dashed blue), and $N=4$ (dotted green). For small values of $\gamma$, the ratio is below one (black dashed line), and tends asymptotically to $1 / N$ : In this regime it is more convenient to employ separable states than maximally entangled states. The ratio increases monotonically with $\gamma$ and exceeds one at a threshold value $\gamma_{0}(N)$, which depends on $N$. For asymptotically large $\gamma$, the use of $N$-qubit GHZ states is $N$ times better than the use of $N$ qubits in a separable state. In the lower left panel, a log-log plot of the optimal interaction time as a function of $\gamma$ for separable probes (black), and for an entangled probe with 2 (solid red), 3 (dashed blue), and 4 (dotted green) qubits. In the lower right panel, the ratio between the optimal time for the GHZ state $t_{\mathrm{GHZ}}$ and for the separable state $t_{\mathrm{SEP}}$.

We notice that $\beta_{\gamma}(t)$ is a monotonically increasing function of $t$ with $\beta_{\gamma}(0)=0$ [see Eq. (2)]. Moreover, we have $\beta_{\gamma}(t) \sim$ $\Gamma(t-1 / \gamma)$ for $t \gg 1$. Thus both $H_{\mathrm{N}}^{\mathrm{SEP}}(\gamma, t)$ and $H_{\mathrm{N}}^{\mathrm{GHZ}}(\gamma, t)$ are asymptotically vanishing and show a single maximum, corresponding to different optimal values of the interaction time, $t_{\mathrm{opt}}^{\mathrm{SEP}}$ and $t_{\mathrm{opt}}^{\mathrm{GHZ}}$, respectively. We refer to this maximum value as the maximal QFI for a specific value of $\gamma$. As is apparent from the above equations, the maximization of the QFI involves transcendental equations and must be done numerically. The behavior of $H_{\mathrm{N}}^{\mathrm{SEP}}(\gamma, t)$ and $H_{\mathrm{N}}^{\mathrm{GHZ}}(\gamma, t)$ is depicted in the upper left panel of Fig. 2. The lower panels of the figure show how the optimal time depends on $\gamma$ for the two measurement schemes.

In situations where we can control the interaction time between the probe and the environment, it will be most convenient to set it to the optimal time. Thus, a fair comparison between separable and entangled probes naturally involves the maximal QFI of the two cases. We therefore introduce the QFI ratio as $R_{\mathrm{N}}(\gamma)=H_{\mathrm{N}, \text { max }}^{\mathrm{GHZ}}(\gamma) / H_{\mathrm{N}, \max }^{\mathrm{SEP}}(\gamma)$, and analyze its behavior as a function of $\gamma$ and N. When $R_{\mathrm{N}}(\gamma)>1$, the use of a $N$ qubits in a GHZ state improves estimation compared to the use of $N$ uncorrelated probes, e.g., in a sequential strategy. Figure 2 illustrates the main results: The ratio $R_{\mathrm{N}}(\gamma)$ is larger than one for $\gamma>\gamma_{0}(N)$, where $\gamma_{0}(N)$ is a threshold value that depends on $N$. Moreover, $R_{\mathrm{N}}(\gamma) \rightarrow N$ for $\gamma \gg \gamma_{0}(N)$. This result is enhanced by the fact that, upon substituting $\tilde{\gamma}=\gamma / \Gamma$ and $\tau=\tilde{\gamma} t$, we may show that the quantum signal-to-noise 
ratio (QSNR) $\tilde{\gamma}^{2} H(\tilde{\gamma})$ does not depend on $\Gamma$. This means that, if one is able to control the coupling between the probe and the system, one can always tune $\Gamma$ to achieve a situation where $R_{\mathrm{N}}(\tilde{\gamma})>1$. All the figures are obtained by setting $\Gamma=1$.

Now a question arises: Is the GHZ state $\left|\Psi_{\mathrm{N}}\right\rangle$ the optimal one? Are there (entangled) states that give even higher QFI? The answer to this question cannot be analytic, because one cannot diagonalize analytically a generic density matrix of a multiqubit state. In order to attack this problem, we first notice that the maximum is achieved for an initial pure state [37]. We have thus generated a large number $\left(n=10^{6}\right)$ of random initial pure states, uniformly distributed according to the Haar measure, for different values of $\gamma$ and for $N=2,3,4$. For each random state, the maximal QFI, $H_{\mathrm{N}}^{\mathrm{RND}}(\gamma)$, resulting from the interaction with a common environment has been numerically evaluated using the expression $H(\gamma)=2 \sum_{n m}\left|\left\langle\psi_{m}\left|\partial_{\gamma} \rho_{\gamma}\right| \psi_{n}\right\rangle\right|^{2} /\left(\rho_{n}+\rho_{m}\right)$, where $\rho_{\gamma}=\sum_{n} \rho_{n}\left|\psi_{n}\right\rangle\left\langle\psi_{n}\right|$ is the diagonal form of the density operator after the interaction with the environment. This value is then used to evaluate the corresponding QFI ratio $H_{\mathrm{N}}^{\mathrm{RND}}(\gamma) / H_{\mathrm{N}}^{\mathrm{SEP}}(\gamma)$, and to compare the estimation precision to the precision achievable using $N$ independent qubits interacting with separate environments. Our results indicate that, for $\gamma \gtrsim \gamma_{0}$, that is, in the region where entanglement is convenient, the GHZ state is indeed the optimal one, thus showing that entanglement is a resource for the estimation of the spectral width of Gaussian noise.

Below the threshold the GHZ state interacting with a common environment is no longer optimal and the optimal strategy involves separable probes interacting with independent environments. For completeness, we anyway look for the optimal state in a common environment and found numerically that the extremal state lies in the same family of states that had been identified in Ref. [40] as optimal probes to improve frequency estimation. The states of this family, for $N$ qubits, have the form $\left|\Phi_{\mathrm{N}}\right\rangle=\sum_{k=0}^{\left\lfloor\frac{1}{2} \mathrm{~N}\right\rfloor} a_{k}|k\rangle$, where $a_{k}$ are normalized real coefficients, $|k\rangle$ is an equally weighted superposition of all $N$-qubit states with a number $k$ or a number $N-k$ of excitations, and $\lfloor\cdot\rfloor$ denotes the integer part. The GHZ state belongs to this family with $a_{0}=1 / \sqrt{2}$ and all other coefficients set to 0 .

Figure 3 illustrates our numerical results obtained for two and three qubits. The plots show the QFI ratios $R_{2}$ (left) and $R_{3}$ (right). The solid blue line is the ratio for the GHZ state, the $10^{4}$ gray points correspond to the QFI ratio of randomly generated states, and the dashed red line is found by optimizing the QFI over the coefficients $a_{k}$ of $\left|\Phi_{\mathrm{N}}\right\rangle$. We can see that from $\gamma \gtrsim \gamma_{0}(N)$ the blue and red curves coincide, i.e., GHZ states are extremal. We also notice that for $\gamma \gtrsim \gamma_{0}(N)$ a significant fraction of gray dots lies above the $r=1$ dashed line, but the dots are sparse around the solid blue line, meaning that the GHZ state allows for a remarkable gain in the estimation of larger values of $\gamma$.

It is worth to emphasize that optimal precision, i.e., the QFI of Eq. (6), may be achieved upon implementing a simple rank-2 measurement. Indeed, $H_{\mathrm{N}}^{\mathrm{GHZ}}(\gamma)$ corresponds to the Fisher information of a projective measurement on the two eigenvectors corresponding to the nonzero eigenvalues of the
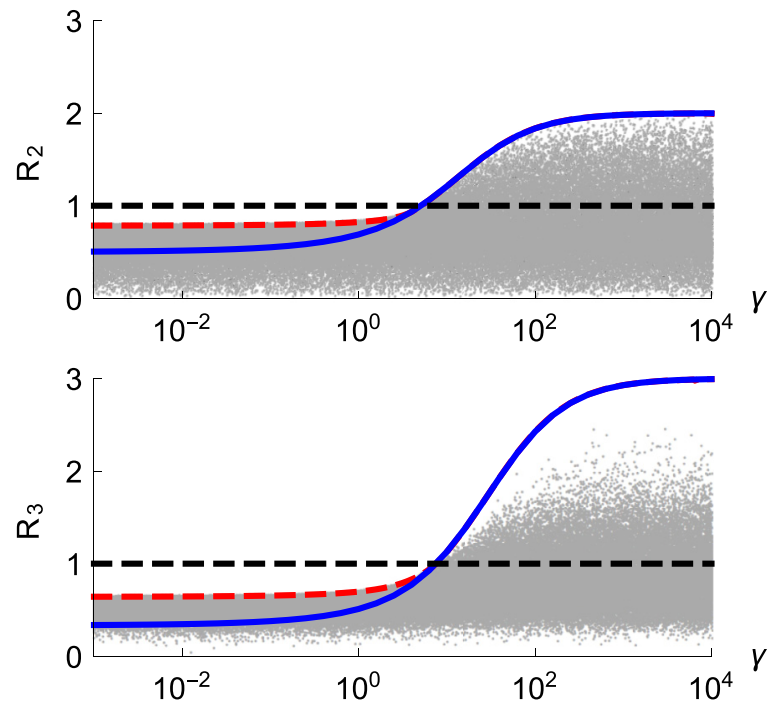

FIG. 3. (Color online) The curves in the log plots show the QFI ratio $R_{2}$ (top) and $R_{3}$ (bottom) as a function of $\gamma$ for the GHZ state (solid blue) and the optimal state of $N$ qubits in a common environment (dashed red). The two curves superimpose each other above the threshold $\gamma_{0}(N)$. We also show the QFI ratio for $10^{5}$ randomly generated states (gray points), uniformly distributed according to the Haar measure.

evolved density operator, which are, respectively,

$$
\begin{gathered}
p_{ \pm}=\frac{1}{2}\left(1 \pm e^{-2 N^{2} \lambda^{2} \beta_{\gamma}(t)}\right) \\
\left|p_{ \pm}\right\rangle=\frac{1}{\sqrt{2}}\left(e^{-i \omega_{0} \sigma_{z}}\right)^{\otimes N}\left(|0\rangle^{\otimes N} \pm|1\rangle^{\otimes N}\right) .
\end{gathered}
$$

The CR theorem, however, sets only a lower bound to the precision of any unbiased estimator, and a question arises on how to suitably process data coming from the above rank-2 measurement in order to saturate the bound. Bayesian estimators are known to saturate the CR bound for asymptotically large numbers of measurements: In order to assess quantitatively the performance of Bayesian estimation we have performed simulated experiments on the probing system. In particular, we have simulated the outcomes $\left\{x_{1}, \ldots, x_{M}\right\}$ of the measurement by randomly choosing a result according to the probabilities of Eq. (7), and have built a Bayesian estimator $\hat{\gamma}$ as the mean value of the a posteriori distribution, starting from a flat prior. The resulting relative error of $\hat{\gamma}, \epsilon=\sqrt{\operatorname{Var}(\hat{\gamma})} / \hat{\gamma}$, is shown, as a function of the number of measurements, in Fig. 4 for a specific value of $\gamma$. We see that with a relatively low number of measurements, of the order of thousands, the bound is saturated and the situation improves by increasing the number of qubits. The proposed scheme thus allows for an effective and achievable estimation of the parameter $\gamma$.

Let us now address the robustness of our scheme against noise in the preparation of the probe. In fact, we have shown that the use of entangled qubit probes prepared in a GHZ state leads to enhanced precision in the estimation of the spectral width. However, it is generally challenging to experimentally prepare the probes exactly in the GHZ state, and a question arises on how sensible is this estimation scheme 


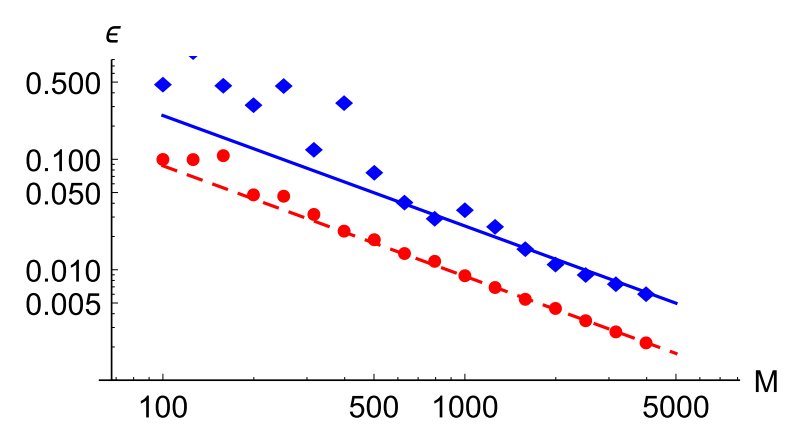

FIG. 4. (Color online) Log-log plot of the relative error $\epsilon=$ $\sqrt{\operatorname{Var}(\hat{\gamma})} / \hat{\gamma}$ of the Bayesian estimator as a function of the number of measurements, for $\gamma=10$. The lines represent the CR bound for a single-qubit measurement (dashed blue) and for a four-qubit GHZ state (solid red). The diamonds (single qubit) and the dots (four qubits) correspond to the performance of a Bayes estimator applied to simulated experiments. Bayes estimators saturate the CR bound when increasing the number of measurements and very good performances are achieved already for thousands of measurements. The plot also shows that estimation improves with the number of qubits since the $\mathrm{CR}$ bound is saturated with a lower number of measurement.

to, e.g., the purity of the initial preparation. We answer this question by considering a partially depolarized state, $\rho_{p}=$ $p \rho_{\mathrm{GHZ}}+(1-p) \mathbb{I} / 2^{N}$, where $\mathbb{I}$ is the identity matrix and $0<$ $p<1$, and a partially dephased state, $\rho_{\delta}=\delta \rho_{\mathrm{GHZ}}+\frac{1}{2}(1-$ $\delta)\left(|0\rangle^{\otimes N}\langle 0|+| 1\rangle^{\otimes N}\langle 1|\right)$, where $0<\delta<1$. In both cases, an analytic expression for the QFI may be found: We have

$$
\begin{gathered}
H_{\mathrm{N}}^{p}(\gamma, t)=\frac{2^{N+2} N^{4}\left[\left(2^{N}-2\right) p+2\right] p^{2}\left[\partial_{\gamma} \beta_{\gamma}(t)\right]^{2}}{\left[\left(2^{N}-2\right) p+2\right]^{2} e^{4 N^{2} \beta(t, \gamma)}-4^{N} p^{2}}, \\
H_{\mathrm{N}}^{\delta}(\gamma, t)=\frac{4 N^{4} \delta^{2}}{e^{4 N^{2} \beta(t, \gamma)}-\delta^{2}}\left[\partial_{\gamma} \beta_{\gamma}(t)\right]^{2},
\end{gathered}
$$

which are obviously less than $H_{\mathrm{N}}^{\mathrm{GHZ}}(\gamma)$, being $\rho_{p}$ and $\rho_{\delta}$ mixed states, but may be still larger than $H_{\mathrm{N}}^{\mathrm{SEP}}(\gamma)$. Indeed, Fig. 5 shows that for each value of $\gamma$ above $\gamma_{0}(N)$ there is a threshold value for the purity, above which the use of a depolarized or dephased GHZ state still leads to an improvement over the use of $N$ uncorrelated probes. The threshold purity $\mu_{0}$ is close to one for $\gamma \simeq \gamma_{0}(N)$ and for $\gamma \gg \gamma_{0}(N)$, whereas it shows a minimum in the intermediate region, thus allowing for a certain tolerance in the preparation of the initial state of the probe. Besides, this minimum value of the threshold gets lower when increasing the number of qubits.

In conclusion, we have shown that the use of entangled qubits as quantum probes outperforms the sequential use of
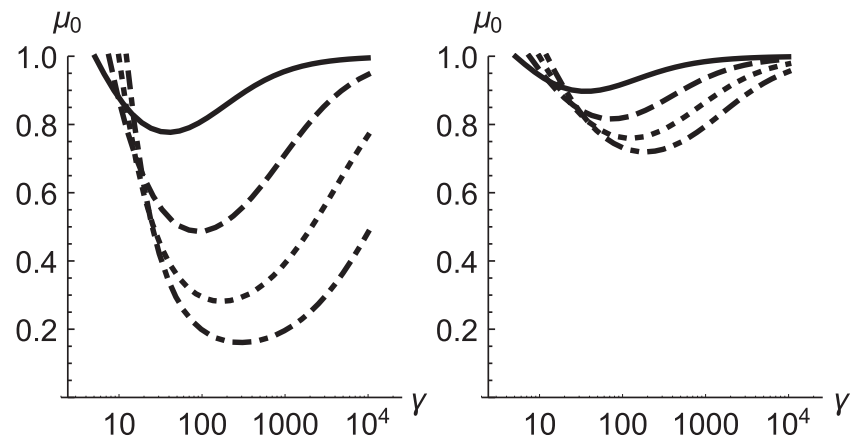

FIG. 5. Threshold purity $\mu_{0}$, as a function of the spectral width $\gamma$, above which the use of a depolarized (left panel) or a dephased GHZ state (right panel) is more effective than a set of uncorrelated qubits. The different lines correspond to a different number $N$ of qubits: $N=2$ (solid), $N=3$ (dashed), $N=4$ (dotted), $N=5$ (dotted-dashed). The threshold $\mu_{0}$ approaches 1 when $\gamma \rightarrow \gamma_{0}(N)$ and when $\gamma \rightarrow \infty$, whereas there is an intermediate region where $\mu_{0}$ decreases to a minimum, meaning that there is more tolerance in the initial preparation of the probe. When $N$ increases, the minimum of $\mu_{0}$ decreases and moves to larger values of $\gamma$.

single-qubit probes in the characterization of the noise induced by complex environments. In particular, we have shown that a joint measurement on entangled probes improves estimation of the correlation time for a broad class of environmental noises when the noise is faster than a threshold value. This result is enhanced by the fact that, upon controlling the coupling between the probe and the system, the threshold value can be reduced arbitrarily. Our scheme exploits timedependent sensitivity of quantum systems to decoherence and does not require dynamical control on the probes. We have found the optimal interaction time and the optimal multiqubit probe preparation, showing that it corresponds to multiqubit GHZ states. The proposed measurement scheme achieves the Cramér-Rao bound for a relatively low number of measurements, upon employing a Bayesian estimator, and is robust against imperfect preparation of the initial entangled state.

This work has been supported by MIUR through the FIRB project "LiCHIS" (Grant No. RBFR10YQ3H), by EU through the Collaborative Project QuProCS (Grant Agreement No. 641277), and by UniMI through the H2020 Transition Grant No.15-6-3008000-625. M.G.A.P. thanks Claudia Benedetti and Rafal Demkowicz-Dobrzanski for discussions.
[1] E. B. Davies, Quantum Theory of Open Systems (Academic Press, New York, 1976).

[2] H.-P. Breuer and F. Petruccione, The Theory of Open Quantum Systems (Oxford University Press, Oxford, UK, 2007).

[3] B. M. Escher, R. L. de Matos Filho, and L. Davidovich, Nat. Phys. 7, 406 (2011).

[4] S. Alipour, M. Mehboudi, and A. T. Rezakhani, Phys. Rev. Lett. 112, 120405 (2014).
[5] P. Hänggi and P. Jung, in Advanced Chemical Physics, edited by I. Prigogine and S. A. Rice (Wiley, Hoboken, NJ, 1995), Vol. 89, pp. 239-326.

[6] J. M. Taylor, P. Cappellaro, L. Childress, L. Jiang, D. Budker, P. R. Hemmer, A. Yacoby, R. Walsworth, and M. D. Lukin, Nat. Phys. 4, 810 (2008).

[7] A. K. Mittermaier and L. E. Kay, Trends Biochem. Sci. 34, 601 (2009). 
[8] S. Zhong and H. Xin, Chem. Phys. Lett. 333, 133 (2001).

[9] J. Bylander, S. Gustavsson, F. Yan, F. Yoshihara, K. Harrabi, G. Fitch, D. G. Cory, Y. Nakamura, J.-S. Tsai, and W. D. Oliver, Nat. Phys. 7, 565 (2011).

[10] J. Zhang, X. Peng, N. Rajendran, and D. Suter, Phys. Rev. A 75, 042314 (2007).

[11] M. A. Ali Ahmed, G. A. Álvarez, and D. Suter, Phys. Rev. A 87, 042309 (2013).

[12] I. Almog, Y. Sagi, G. Gordon, G. Bensky, G. Kurizki, and N. Davidson, J. Phys. B 44, 154006 (2011).

[13] L. Banchi, P. Giorda, and P. Zanardi, Phys. Rev. E 89, 022102 (2014).

[14] R. Vasile, F. Galve, and R. Zambrini, Phys. Rev. A 89, 022109 (2014).

[15] A. W. Chin, S. F. Huelga, and M. B. Plenio, Phys. Rev. Lett. 109, 233601 (2012).

[16] P. Haikka and S. Maniscalco, Open Syst. Inf. Dyn. 21, 1440005 (2014).

[17] M. G. A. Paris, Int. J. Quantum Inf. 7, 125 (2009).

[18] G. A. Álvarez and D. Suter, Phys. Rev. Lett. 107, 230501 (2011).

[19] M. G. A. Paris, Physica A 413, 256 (2014).

[20] C. Benedetti, F. Buscemi, P. Bordone, and M. G. A. Paris, Phys. Rev. A 89, 032114 (2014).

[21] Ł. Cywiński, Phys. Rev. A 90, 042307 (2014).

[22] C. Benedetti and M. G. A. Paris, Phys. Lett. A 378, 2495 (2014).

[23] D. Crow and R. Joynt, Phys. Rev. A 89, 042123 (2014).

[24] I. Neder, M. S. Rudner, H. Bluhm, S. Foletti, B. I. Halperin, and A. Yacoby, Phys. Rev. B 84, 035441 (2011).
[25] M. J. Biercuk and H. Bluhm, Phys. Rev. B 83, 235316 (2011).

[26] W. M. Witzel, K. Young, and S. Das Sarma, Phys. Rev. B 90, 115431 (2014).

[27] T. Fink and H. Bluhm, arXiv:1402.0235.

[28] T. Yu and J. Eberly, Opt. Commun. 283, 676 (2010).

[29] J.-Q. Li and J.-Q. Liang, Phys. Lett. A 375, 1496 (2011).

[30] C. Benedetti, F. Buscemi, P. Bordone, and M. G. A. Paris, Phys. Rev. A 87, 052328 (2013).

[31] C. Benedetti, F. Buscemi, P. Bordone, and M. G. A. Paris, Int. J. Quantum Inf. 10, 1241005 (2012).

[32] J. Li, M. P. Silveri, K. S. Kumar, J.-M. Pirkkalainen, A. Vepsäläinen, W. C. Chien, J. Tuorila, M. A. Sillanpää, P. J. Hakonen, E. V. Thuneberg, and G. S. Paraoanu, Nat. Commun. 4, 1420 (2013).

[33] G. Burkard, Phys. Rev. B 79, 125317 (2009).

[34] H. J. Wold, H. Brox, Y. M. Galperin, and J. Bergli, Phys. Rev. B 86, 205404 (2012).

[35] C. Benedetti, M. G. A. Paris, and S. Maniscalco, Phys. Rev. A 89, 012114 (2014).

[36] G. M. D'Ariano, P. Lo Presti, and M. G. A. Paris, Phys. Rev. Lett. 87, 270404 (2001).

[37] A. Fujiwara, Phys. Rev. A 63, 042304 (2001).

[38] A. Acín, E. Jané, and G. Vidal, Phys. Rev. A 64, 050302 (2001).

[39] See Supplemental Material at http://link.aps.org/supplemental/ 10.1103/PhysRevA.92.010302 for a detailed description with analytic formulas of the four cases described in the article.

[40] S. F. Huelga, C. Macchiavello, T. Pellizzari, A. K. Ekert, M. B. Plenio, and J. I. Cirac, Phys. Rev. Lett. 79, 3865 (1997). 